\title{
Clinicopathological Evaluation of Clear Cell Hidradenoma (Acrospiroma) Within Multiple Tumor Complex in a Dog
}

\author{
Mehmet Eray Alcigir', Tuncer Kutlư ${ }^{2}$, Irem Ergin ${ }^{3} \&$ Gonca Sonmez ${ }^{3}$
}

\begin{abstract}
Background: Clear cell hidradenoma (acrospiroma) is adnexal tumors that arise from the distal excretory duct of eccrine sweat glands. It is generally defined in humanbeings. It presents solitary structure in firmness nodule. Most frequently are encountered in head, face, and upper extremities in humans. Hidradenomas are called generally by definition benign. Their malignant transformations and metastasis are seen very rarely. In veterinary literature database, the malignant form of tumor has been defined as an unique report in four dogs. They are extremely rare tumors in dogs. It is not documented within multiple tumor complex up to now.

Case: In the case, three tumors were detected in a 9-year-old neutered female German shepherd dog. After clinical examination, a complete surgical resection of all masses was performed and masses were sent to Pathology for diagnosis. A mass on the right last rib was a $8 \times 6 \mathrm{~cm}$ in diameters with severe ulceration. The mass had a hard texture, immobility and irregular borders. Other masses were localized on the back and left caudo-abdominal mammary lobe. Tumors on the back and the mammary lobe did not invade muscle layer, but the tumor on the last rib aggressively invaded surrounding tissue and were very difficult to remove surgically After macroscopy, all the masses were stained with hematoxylin-eosin (H\&E), Mayer's mucicarmine and Periodic Acid Shiff (PAS) stainings. In the first mass, there were multilobular epithelial islands between prominent fibrous septa from the upper to deep of dermis. The neoplastic cells were generally round or polyhedral in shape. Some of cytoplasms were eosinophilic at different degree. But cytoplasms were generally finely granular and vesicular or clear in appearance. Some cells had possible glycogen deposits. Nucleus was oval to round and had fine reticular chromatin and a distinct nucleolus. The tumor was diagnosed as clear cell hidradenoma. Others were was belonged to hemangioma on the back and malignant mixed tumor in the mammary gland.

Discussion: In our case, two cell types having benign characteristics were also noted. Some clear cells contained PAS (+) material showing glycogen deposits. Additionally, there were some fibrous septa separating those cell islands. Thus, it is thought that the histopathology shows parallelism to many reports described in human beigns. Clear cell hidradenoma described in this case is first documentation as benign counterpart on the basis of veterinary literatures even though a previous report including clear cell hidradenocarcinoma in four dogs. And also, it has been reported to be very rarely seen tumor in human beings. However, any hormonies in terms of its predilection site can not be found among the cases with clear cell hidradenoma in human counterparts, because the tumor is encountered at skin of last right rib. This situation has shown us the tumor does not select any predilection site as described in this case. It has been understood on the basis of literatures that this is the first case report of describing benign tumor of ecrine sweat duct in dogs. Also, there has been no any documentation regarding in this multiple tumor complex.
\end{abstract}

Keywords: clear cell hidradenoma, dog, multiple tumor complex. 


\section{INTRODUCTION}

Clear cell hidradenoma or acrospiroma has histologically distinctable benign characteristics and highly rare tumors of sweat gland duct origin. It is generally defined in humanbeings. It presents solitary structure in firmness nodule. Most frequently are encountered in head, face, and upper extremities in humans. Although traditionally regarded as displaying eccrine differentiation, it is now accepted that tumors can show either eccrine or apocrine differentiation [1-3]. Hidradenomas are called generally by definition benign. Their malignant transformations and metastasis are seen very rarely [3]. In veterinary literature database, the malignant form of tumor has been defined as an unique report in four dogs [4]. In acrospiroma, two main types of cells were seen. One cell type is polyhedral with a rounded nucleus and slightly basophilic cytoplasm. The second cell type is generally round with clear cytoplasm [5]. Thus, further analysis is not important in diagnosis, because such cases can be detected easily in routine Hematoxyline \& Eosin stainings.

\section{CASE}

A 9-year-old neutered female German shepherd dog was reffered to clinic in Ankara University, Faculty of Veterinary Medicine with complaints of rapidly grown of three tumors on its different parts of body. After clinical examination, a complete surgical resection of all mass was performed with the permissions of the dog's owner and masses were sent to Pathology for diagnosis. After macroscopy, routin histological staining procedure was performed. Serial tissue samples from all the masses were stained with Hematoxylin-Eosin (H\&E), Mayer's mucicarmine and Periodic Acid Shiff (PAS) stainings.

The masses on the right last rib was a $8 \times 6 \mathrm{~cm}$ in diameters with severe ulceration. The mass had a hard texture, immobility and irregular borders. Other masses were localized on the back and left caudo-abdominal mammary lobe. Tumors on the back and the mammary lobe did not invade muscle layer, but the tumor on the last rib aggressively invaded surrounding tissue and were very difficult to remove surgically (Figure 1 A-C). In the first mass, there were multilobular epithelial islands between prominent fibrous septa from the upper to deep of dermis. The neoplastic cells were generally round or polyhedral in shape. Some of cytoplasms were eosinophilic at different degree. But cytoplasms

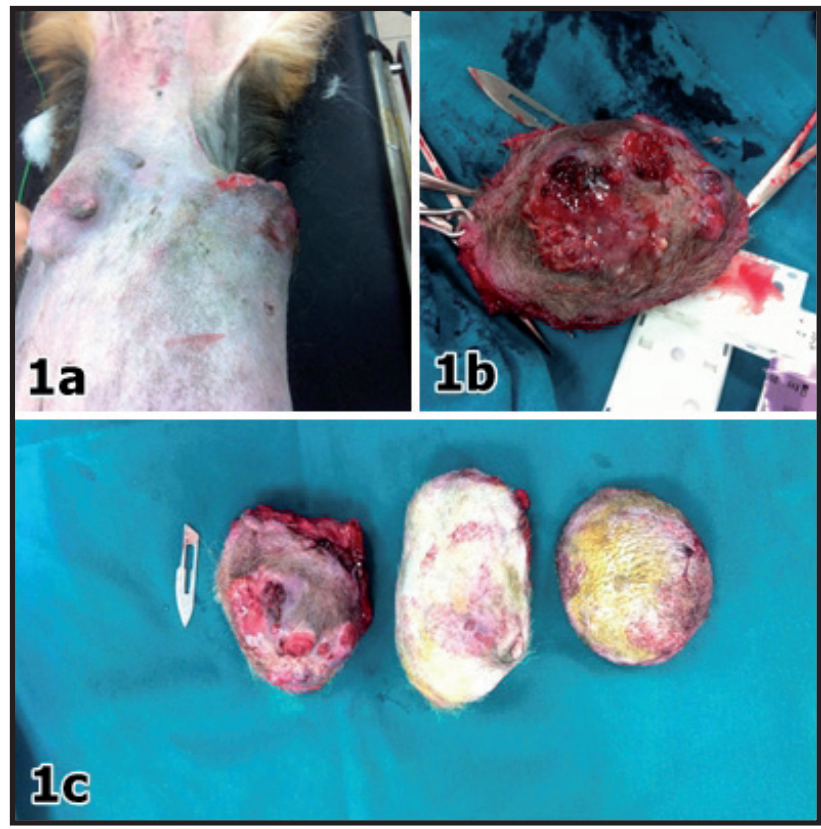

Figures 1. A- A mass localized at left caudo-abdominal mammary lobe (upside) and another ulcerated mass localizated on the last rib (downside) noted during clinical examinations. B- Appearence of the ulcerated mass on the last right rib with haemorrhagic areas after its surgical excision. C- Macroscopical views of all masses excised surgically.

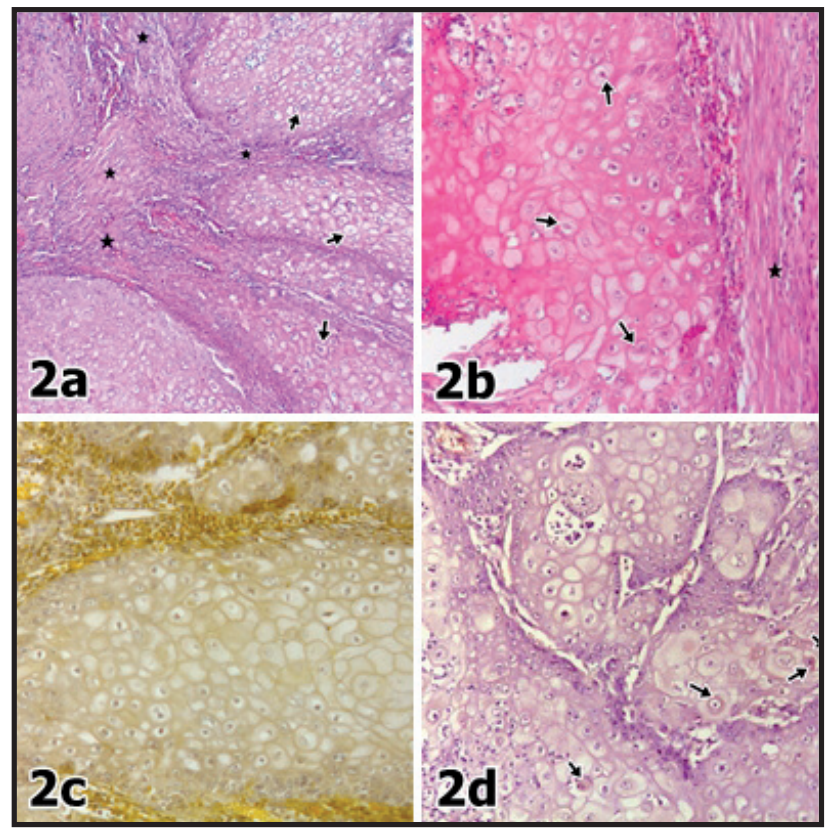

Figures 2. A- Clear cell islands of acrospiroma (arrows) and fibrous septa(stars) [H\&E, 40X]. B- Clear cells in polyhedral shape with large nuclei of acrospiroma (arrows) and bundles of fibrocytes (stars) [H\&E, 100X]. C- Mucicarmine negativity in clear cells of acrospiroma, 100x. D- Periodic Acid Shiff staining positivities in clear cells of acrospiroma (arrows) [100x].

were generally finely granular and vesicular or clear in appearance. Some cells had possible glycogen deposits. Nucleus was oval to round and had fine reticular chromatin and a distinct nucleolus. The tumor was diagnosed as clear cell hidradenoma (Figure 2 A-D). Others were 


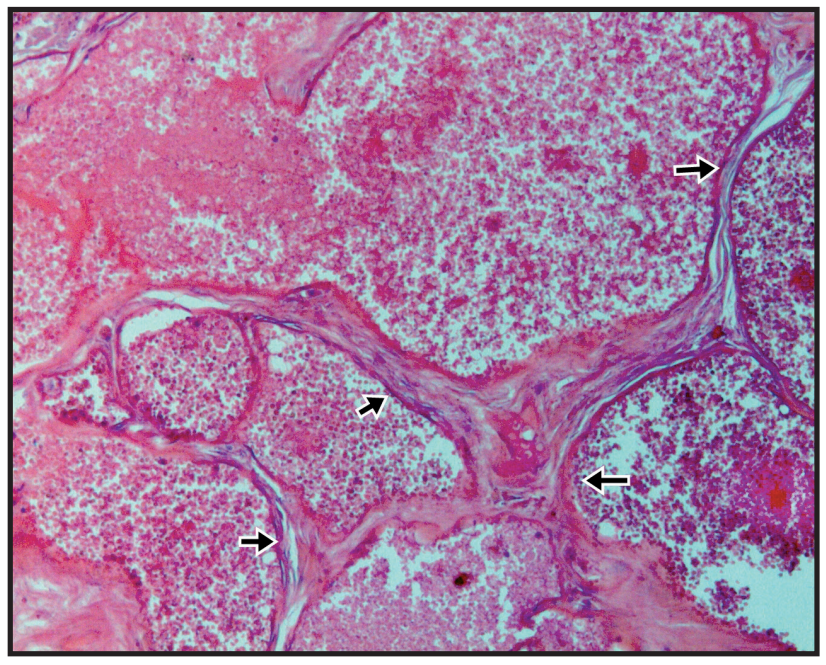

Figure 3. Neoplastic vessels lining by uniform endothelial cells (arrows) in hemangioma [H\&E, 100X].

belonged to hemangioma on the back and malignant mixed tumor in the mammary gland (Figures 3-4). The slides were examined (Olympus CX31) ${ }^{1}$ and photographed (Olympus DP12) ${ }^{1}$ using a light microscope.

\section{DISCUSSION}

Clear cell hidradenoma includes a polyhedral shape with a rounded nucleus and round shape with clear cytoplasm [5]. In our case, two cell types having benign characteristics were also noted. Some clear cells contained PAS (+) material showing glycogen deposits. Additionally, there were some fibrous septa separating those cell islands. Thus, it is thought that the histopathology shows parallelism to many reports described in human beigns. On the other side, clear cell hidradenoma described in this case is first documentation as benign counterpart on the basis of veterinary literatures even though a previous report including clear cell hidradenocarcinoma in four dogs [4]. And also, it has been reported to be very rarely seen tumor in human beings [3]. However, any hormonies in terms

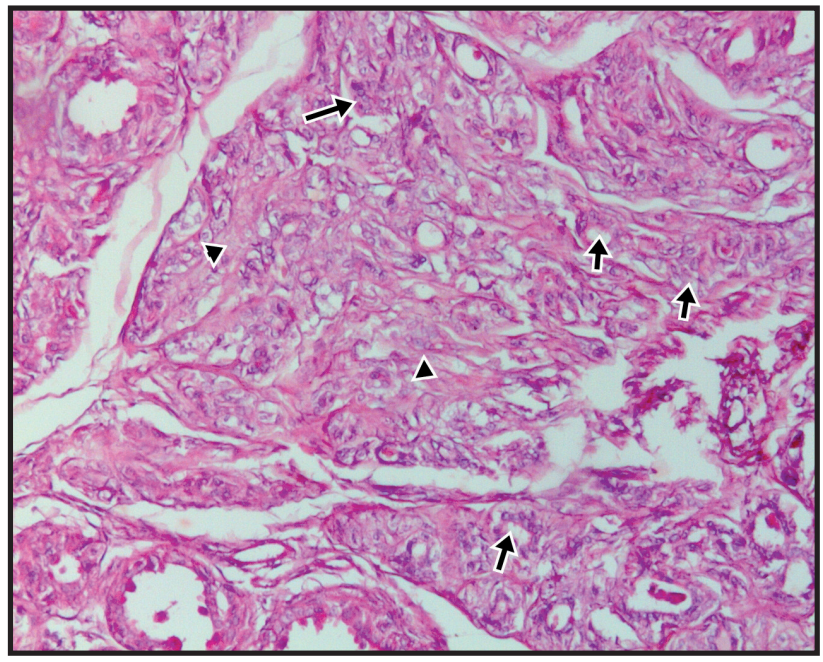

Figure 4. Anaplastic mammary epithelial cells trying to gland pattern (arrows) in fibrous matrix (arrow heads) [H\&E, 100X].

of its predilection site can not be found among the cases with clear cell hidradenoma in human counterparts [3], because the tumor is encountered at skin of last right rib. This situation has shown us the tumor does not select any predilection site as described in this case.

To our knowledge, this is the first case of a clear cell hidradenoma in a dog. Also this case is found interestingly due to having different localization and origin of tumors in the same dog. It has been believed that this presentation will be usefull to researchers which will study about skin adnexal tumors' clinicopathology.

\section{MANUFACTURER}

${ }^{1}$ Olympus Corporation. Tokyo, Japan.

Acknowledgements. The case report were presented as poster presentation in European Veterinary Conference Voorjaarsdagen 2017 in Netherland.

Declaration of interest. The authors report no conflicts of interest. The authors alone are responsible for the content and writing of the paper.

\section{REFERENCES}

1 Gianotti F. \& Alessi E. 1997. Clear cell hidradenoma associated with the folliculo- sebaceous apocrine unit. Histologic study of five cases. The American Journal of Dermatopathology. 19: 351-357.

2 Hashimoto K., Bella R. \& Level W. 1967. Clear cell hidradenoma: Histologic, histochemical and electron microscopic study. Archives of Dermatology. 96: 18-38.

3 Hernández-Pérez E. \& Cestoni-Parducci R. 1985. Nodular hidradenoma and hidradenocarcinoma. A 10-year review. Journal of the American Academy of Dermatology. 12(1): 15-20.

4 Jabara A.G. \& Finnie J.W. 1978. Four cases of clear-cell hidradenocarcinomas in the dog. Journal of Comparative Pathology. 88(4): 525-532.

5 Weedon D. 2002. Tumors of cutaneous appendages. In: Skin Pathology. 2nd edn. London: Churchill Livingston, pp.859-916. 